in 25 Patients (46.3\%of cases). Anti-citrullinated peptide antibody (ACPA) was positive in 32 patients (59.3\%).41patients (75.4\%) had radiological impairments and $28(51.9 \%)$ had specific deformations of RA. The average disease activity score (DAS28-VS) and (DAS28-CRP) were respectively 4.1 $1.5[1.4-7.3]$ $3.4 \pm 1.5$ [1.24-6.71]. Oral examination revealed a poor oral hygiene in 36patients ( $69.2 \%$ of cases) and $4.7 \%$ of our patients (2 cases) were toothless. Xerostomia was observed in 32 patients $(80 \%)$. Gingivitis was diagnosed in 26 Patients (52\%): localized in 6 patients (26.1\%) and generalized in 17 patients $(73.9 \%) .21$ patients had periodontitis (41.2\%). Basing on bleeding index of Loe and Silness (IG),27 patients (55.1\%) had degree 2 and9 patients (18.8\%) had degree 3 . Supragingival plaque and subgingival plaque were detected respectively in 45 patients $(90 \%)$ and 47 patients $(95.9 \%)$. In our study, tooth loss was significantly correlated with increased age $(p=0.001)$ and post-menopausal status $(p=0.03)$. Xerostomia, gingivitis and periodontitis were associated with increased age. But no association was found between oral manifestations and DAS28 nor biological inflammatory parameters.

Conclusion: Rheumatoid arthritis is destructive and disabling rheumatism with a great risk to develop dental and periodontal diseases. So, it is important to systematically control oral hygiene of our patients to prevent complications.

Disclosure of Interests: None declared

DOI: 10.1136/annrheumdis-2021-eular.4015

\section{POS0586 RHEUMATOID ARTHRITIS IS AN INFLAMMATORY DISEASE WITH A HIGH CARDIOVASCULAR RISK}

L. Nacef ${ }^{1}$, H. Riahi ${ }^{2}$, Y. Mabrouk ${ }^{1}$, H. Ferjani ${ }^{3}$, K. Maatallah ${ }^{3}$, E. Labbene ${ }^{4}$, D. Kaffel ${ }^{1}$, M. Bouaziz ${ }^{2}$, W. Hamdi ${ }^{1} .{ }^{1}$ Mohamed Taieb Kassab Institute of Orthopedic Surgery and Traumatology, Rheumatolgy, Manouba, Tunisia; ${ }^{2}$ Mohamed Taieb Kassab Institute of Orthopedic Surgery and Traumatology, Radiology, Tunis, Tunisia; ${ }^{3}$ Mohamed Taieb Kassab Institute of Orthopedic Surgery and Traumatology, Rheumatolgy, Tunis, Tunisia; ${ }^{4}$ Mohamed Taieb Kassab Institute of Orthopedic Surgery and Traumatology, Radiology, Manouba, Tunisia

Background: Hypertension, diabetes, and dyslipidemia are traditional risk factors of cardiac events. Carotid ultrasonography is an available way to detect subclinical atherosclerosis

Objectives: This study aimed to compare the intima-media thickness in RA patients based on their personal cardiovascular (CV) history of hypertension (hypertension), diabetes, and dyslipidemia.

Methods: The present study is a prospective study conducted on Tunisian RA patients in the rheumatology department of Mohamed Kassab University Hospital (March and December 2020). The characteristics of the patients and those of the disease were collected.

The high-resolution B-mode carotid US measured the IMT, according to American Society of Echocardiography guidelines. The carotid bulb below its bifurcation and the internal and external carotid arteries were evaluated bilaterally with grayscale, spectral, and color Doppler ultrasonography using proprietary software for carotid artery measurements. IMT was measured using the two inner layers of the common carotid artery, and an increased IMT was defined as $\geq 0.9 \mathrm{~mm}$. A Framingham score was calculated to predict the cardiovascular risk at 10 -year.

Results: Forty-seven patients were collected, $78.7 \%$ of whom were women. The mean age was $52.5 \pm 11.06$ [32-76]. The rheumatoid factor (RF) was positive in $57.8 \%$ of cases, and anti-citrullinated peptide antibodies (ACPA) were positive in $62.2 \%$ of cases. RA was erosive in $81.6 \%$ of cases. Hypertension (hypertension) was present in $14.9 \%$ of patients, diabetes in $12.8 \%$ of patients, and dyslipidemia in $12.8 \%$ of patients. Nine patients were active smokers. The mean IMT in the left common carotid (LCC) was $0.069 \pm 0.015$, in the left internal carotid (LIC) was $0.069 \pm 0.015$, in the left external carotid (LEC) was $0.060 \pm 0.023$. The mean IMT was $0.068 \pm 0.01$ in the right common carotid (RCC), $0.062 \pm 0.02$ in the right internal carotid $(\mathrm{RIC})$, and $0.060 \pm 0.016$ in the right external carotid (REC). The IMT was significantly higher in the left common carotid (LCC) in patients with hypertension $(p=0.025)$. There was no significant difference in the other ultrasound sites (LIC, LEC, RCC, RIC, and REC) according to the presence or absence of hypertension. The IMT was also significantly increased in patients with diabetes at LCC $(p=0.017)$ and RIC $(p=0.025)$. There was no significant difference in the IMT at different ultrasound sites between patients with and without dyslipidemia.

Conclusion: Hypertension was significantly associated with the increase in IMT at the LCC level in RA patients. Diabetes had an impact on IMT in LCC and RIC. However, dyslipidemia did not affect the IMT at the different ultrasound sites. REFERENCES:

[1] S. Gunter and al. Arterial wave reflection and subclinical atherosclerosis in rheumatoid arthritis. Clinical and Experimental Rheumatology 2018; 36: Clinical E.xperimental.

[2] Aslan and al. Assessment of local carotid stiffness in seronegative and seropositive rheumatoid arthritis. SCANDINAVIAN CARDIOVASCULAR JOURNAL, 2017.
[3] Martin I. Wah-Suarez and al, Carotid ultrasound findings in rheumatoid arthritis and control subjects: A case-control study. Int J Rheum Dis. 2018;1-7.

[4] Gobbic C and al. Marcadores subclínicos de aterosclerosis y factores de riesgo cardiovascular en artritis temprana. Subclinical markers of atherosclerosis and cardiovascular risk factors in early arthritis marcadores subclínicos de aterosclerose e fatores de risco cardiovascular na artrite precoce.

Disclosure of Interests: None declared

DOI: 10.1136/annrheumdis-2021-eular.4080

\section{\begin{tabular}{|l|l}
\hline POS0587 & EFFECT OF RHEUMATOID ARTHRITIS TREATMENTS
\end{tabular} ON THE INTIMA-MEDIA THICKNESS OF SUPRA- AORTIC TRUNKS AND ON CARDIOVASCULAR RISK}

L. Nacef ${ }^{1}$, H. Ferjani ${ }^{2}$, K. Maatallah ${ }^{1}$, Y. Mabrouk ${ }^{2}$, H. Riahi ${ }^{3}$, E. Labbene ${ }^{4}$

D. Kaffel ${ }^{2}$, W. Hamdi ${ }^{1}$, M. Bouaziz ${ }^{3} .{ }^{1}$ Mohamed Taieb Kassab Institute of

Orthopedic Surgery and Traumatology, Rheumatolgy, Tunis, Tunisia; ${ }^{2}$ Mohamed Taieb Kassab Institute of Orthopedic Surgery and Traumatology, Rheumatolgy, Manouba, Tunisia; ${ }^{3}$ Mohamed Taieb Kassab Institute of Orthopedic Surgery and Traumatology, Radiology, Tunis, Tunisia; ${ }^{4}$ Mohamed Taieb Kassab Institute of Orthopedic Surgery and Traumatology, Radiology, Manouba, Tunisia

Background: Patients with rheumatoid arthritis (RA) are exposed to a multifactorial cardiovascular risk: chronic inflammation, endogenous and exogenous factors, and treatment.

Objectives: The aim of this study was to investigate the impact of RA treatments on cardiovascular risk and the influence of supra aortic trunks.

Methods: This is a prospective study conducted on RA patients followed-up between March and December 2020 at the rheumatology department of the Mohamed Kassab Institute of Orthopedics and Traumatology. Socio-demographic data of patients and characteristics of the disease were collected. The disease activity was evaluated by the Disease Activity Score 28. Prescribed treatments were specified. Ultrasonography of the supra aortic trunks was performed by measuring, in centimeters, the Intima-media Thickness (IMT) at the level of the left (LCC) and right (RCC) common carotid arteries, the left (LIC) and right (RIC) internal carotid arteries and the left (LEC) and right (REC) internal carotid arteries. Results: Of the 47 patients surveyed, $78.7 \%$ were female. The mean age was $52.5 \pm 11.06$ [32-76]. The average RA progressed from $86.25 \pm 63$ [5-288] and was erosive in $81.6 \%$ of cases. The rheumatoid factor (RF) was positive in $57.8 \%$ of patients, and citrullinated antipeptide antibodies (ACPA) were present in $62.2 \%$ The treatments taken were: Methotrexate (MTX) (54.5\%), Sulfasalazine (SLZ) $(1.8 \%)$, Leflunomide (LFN) $(3.6 \%)$, a combination of cs-DMARDs $(5.5 \%)$, and biotherapy $(10.9 \%)$. The prescribed biotherapies were: Etanercept $(3.6 \%)$, Adalimumab (1.8\%), Certolizumab (1.8\%), Infliximab (3.6\%). Corticosteroids (CT) were prescribed in $38.2 \%$ of patients, non-steroidal anti-inflammatory drugs (NSAIDs) (3.6\%), and analgesics (41.8\%).

CT had a protective effect on IMT in LIC $(\mathrm{p}=0.031)$ and RIC $(\mathrm{p}=0.016)$. MTX had a significant protective effect on IMT in RIC $(p=0.002)$ and LEC $(p=0.033)$

SLZ was associated with an increase in IMT at the RIC level $(p=0.05)$. There was no association between NSAID use and IMT. MTX and CT were significantly associated with a decrease in SCORE $(p=0.02 ; p=0.05$, respectively). There was a non-significant association between SLZ or LFN and decreased SCORE $(p=0.140, p=0,970)$

Conclusion: In our series, patients taking MTX and CT had a lower IMT than those not taking these drugs. SLZ was associated with an increase in IMT. NSAIDs did not affect IMT in our study.

REFERENCES:

[1] Pasquale Ambrosino and al, Subclinical atherosclerosis in patients with rheumatoid arthritis A meta-analysis of literature studies. Thrombosis and Haemostasis 113.5/2015

[2] Hyun-Je Kim and al, Effects of Methotrexate on Carotid Intima-media Thickness in Patients with Rheumatoid Arthritis. The Korean Academy of Medical Sciences 2015.

Disclosure of Interests: None declared

DOI: 10.1136/annrheumdis-2021-eular.4120

\section{POS0588 USUAL INTERSTITIAL PNEUMONIA DURING RHEUMATOID ARTHRITIS: PREVALENCE AND ASSOCIATED FACTORS}

C. Daldoul ${ }^{1}$, N. El Amri ${ }^{1}$, A. Guiga ${ }^{2}$, S. Laataoui ${ }^{1}$, K. Baccouche ${ }^{1}$, H. Zegaloui ${ }^{1}$, E. Bouajina ${ }^{1}$. ${ }^{1}$ Farhat Hached Hospital, Rheumatology, Sousse, Tunisia; ${ }^{2}$ Farhat Hached Hospital, Internal Medicine, Sousse, Tunisia

Background: Lung involvement is the second common extraarticular manifes tation of rheumatoid arthritis (RA). Its prevalence varies widely according to the screening tool used and it could reach up to $80 \%$ of patients. This lung disease can affect all the lung compartments. However, interstitial lung disease during RA needs a particular attention due to the increased morbidity and usual interstitial pneumonia (UIP) pattern especially due to its higher rate of mortality. 